Scientific Journal of Hamadan Nursing \& Midwifery Faculty - ISSN 2008-2819

\title{
The Effect of Education Based on BASNEF Model on Introduction of Complementary Feeding
}

Roya Amini', Maryam Madakon², Masoud Khodaveisi ${ }^{3 *}$, Maryam Farhadian ${ }^{4}$

1. Instructor, Chronic Diseases (Home Care) Research Center, School of Nursing and Midwifery, Hamadan University of Medical Sciences, Hamadan, Iran

2. MSc Student of Community Health Nursing, Student Research Committee, School of Nursing and Midwifery, Hamadan University of Medical Sciences, Hamadan, Iran

3. Associated Professor, Chronic Diseases (Home Care) Research Center, Hamadan University of Medical Sciences, Hamadan, Iran

4. Assistant Professor, Department of Biostatistics, School of Public Health, Hamadan University of Medical Sciences, Hamadan, Iran

\begin{tabular}{lll}
\hline Article Info & Abstract \\
\hline
\end{tabular}

Received: $\quad 2017 / 05 / 4$

Accepted: $\quad$ 2017/06/6

Published Online 2017/07/17

DOI: $10.30699 /$ sjhnmf.26.5.4

Original Article

Use your device to scan and read the article online

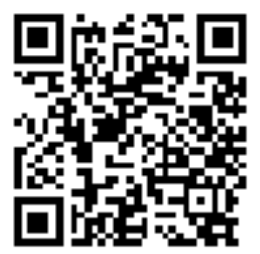

Introduction: The mother has a significant role in introducing food to her child. In present study, the effect of complementary feeding introduction based on BASNEF model on the practice of primiparous mothers was investigated.

Methods: This was a two groups pre\&post test and quasi experimental study carried out on 160 primiparous mothers with 5 months children referred to healthcare centers in Hamadan. Mothers were put into two groups. At first both groups completed a questionnaire which was based on BASNEF model. Then the intervention group was instructed during four sessions (60 min) based on BASNEF model. Two months later, data was collected via questionnaires and a mother performance checklist in two groups again. Data were analyzed by SPSS18 software through Chisquare and Mann-Whitney.

Results: Before the intervention, there was no significant difference in the mean scores of BASNEF structures in the two groups $(P>0.05)$, but after the intervention a significant difference was observed in the mean scores $(P<0.001)$. Statistical tests showed significant differences in the mean scores of BASNEF structures in case group before and after intervention $(P<0.001)$, meanwhile the differences of mean scores of control group was not significant $(P>.0 .05)$. Supplementary feeding time of the intervention and the control groups was $5.88 \pm 0.49$ and $6.14 \pm 0.35$ months respectively. The mean scores of supplementary feeding performance of two groups of mothers, had significant differences $(P<0.001)$.

Conclusion: BASNEF MODEL can be an efficient tool for increasing knowledge, attitude, enabling factors, subjective norms, intention and performance of introduction of complementary feeding of mothers; so we suggest health education programs instead of applying traditional instructions.

Keywords: Complementary Feeding, BASNEF Model, Children.

Copyright (C) 2018, Sci J Hamadan Nurs Midwifery Fac. This is an open-access article distributed under the terms of the Creative Commons Attribution-noncommercial 4.0 International License which permits copy and redistribute the material just in noncommercial usages, provided the original work is properly cited.

How to Cite This Article:

Amini R, Madadkon M, Khodaveisi M, Farhadian M. The Effect of Education Based on BASNEF Model on Introduction of Complementary Feeding. Sci J Hamadan Nurs Midwifery Fac. 2018; 25 (5): 180 - 188 


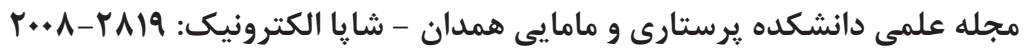

مقاله يزوهشى

\section{تأثير آموزش مبتنى بر مدل بزنف بر نحوهُ شروع تغذيةٌ تكميلى كودكان}

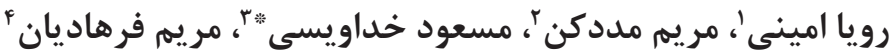

مربى، مركز تحقيقات مراقبت بيمارىهاى مزمن در منزل، دانشكده يرستارى و مامايى، دانشگاه علوم يزشكى همدان، همدان،

دانشجوى كارشناسى ارشد يرستارى سلامت جامعه، كميته تحقيقات دانشجويى، دانشكدهُ يرستارى و مامايى، دانشگاه علوم

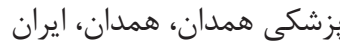

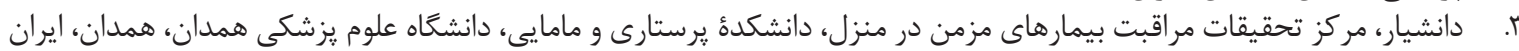

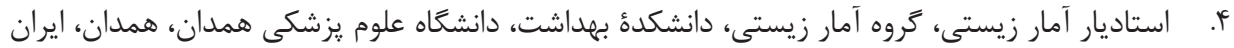

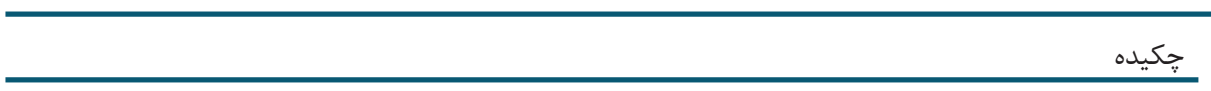

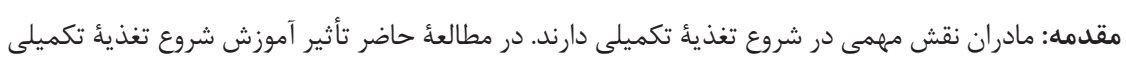

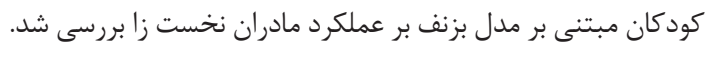

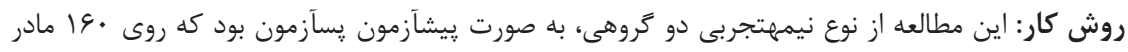

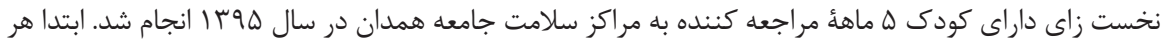

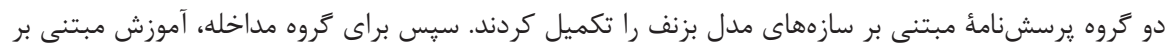

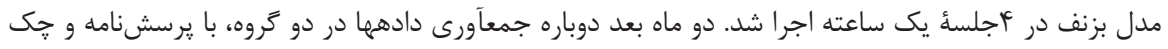

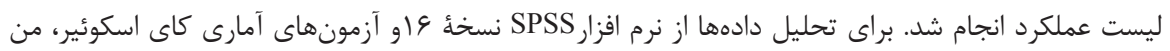

$$
\text { ويتنى و ويلكاكسون استفاده شد. }
$$

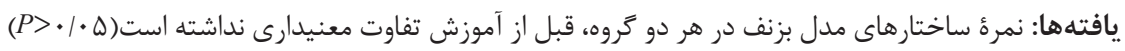

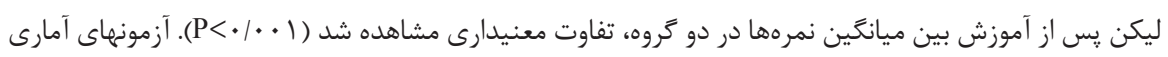

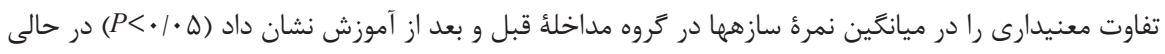

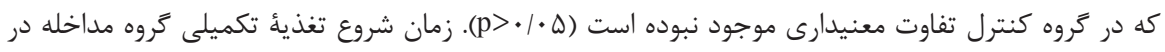

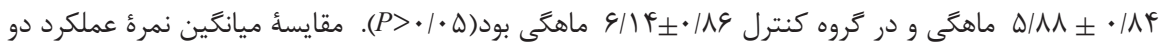

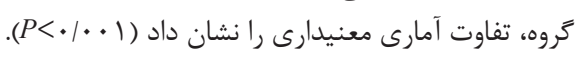
نتيجهَيرى: آموزش مىتواند ابزارى اثربخش براى ارتقاى آَاهى، نحرش، هنجارهاى انتزاعى، عوامل قادر

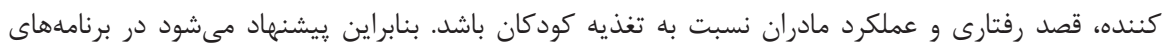

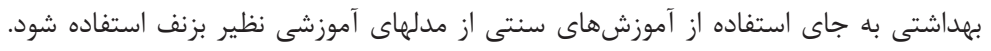

\section{وازههاى كليدى: تغذيأ تكميلى، مدل بزنف، كودكان}

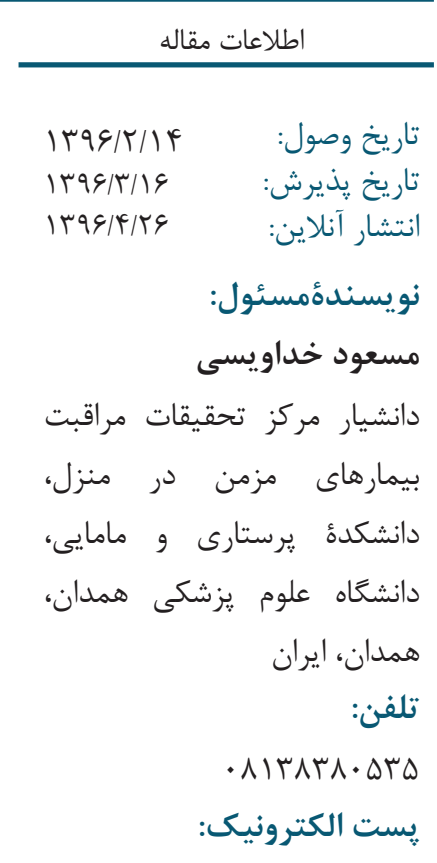

khodaveisi@umsha.ac.ir

$$
\text { آهن، روى و ساير املاح را تأمين كند [ع]. }
$$

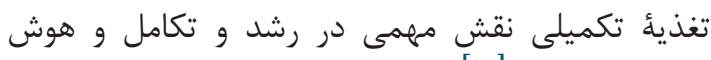

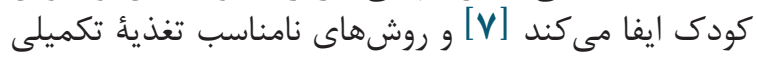

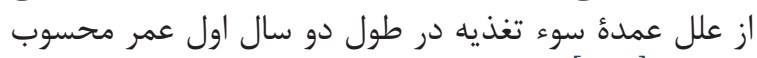

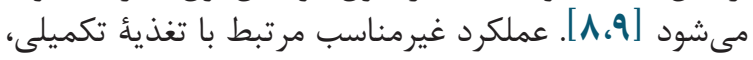

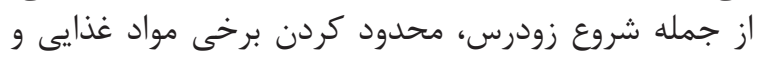

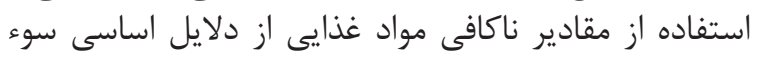

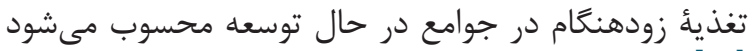

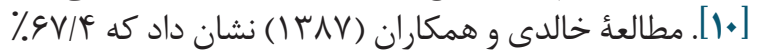

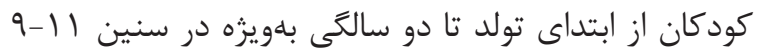

دوره DV شماره D - بهمن و اسفند وqها
مقدمه

تغذيئ صحيح نقش مهمى در تامين سلامتى داشته [1] موانه

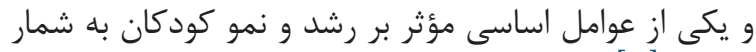

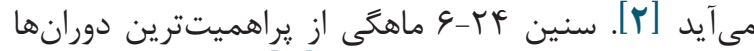

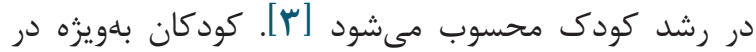
سن شش ماهكَ و با شروع تغذئُ تكميلى بسيار آسيب ميذير

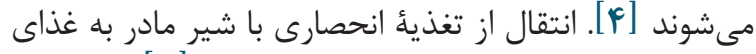

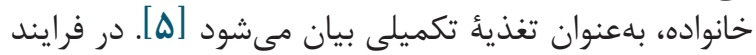

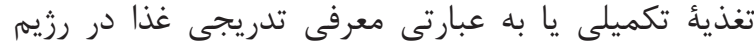

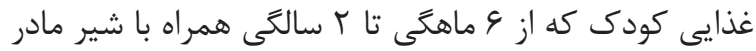

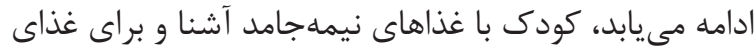

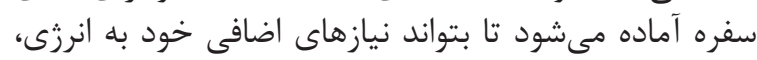
مجله علمى دانشكده يرستارى و مامايى همدان 
مشاهده مىشود كه مادران نخستزا بهدليل كمبود تجربه،

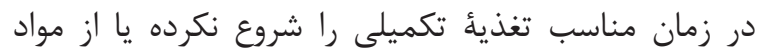

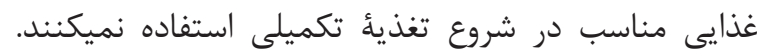

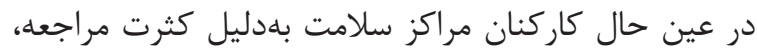

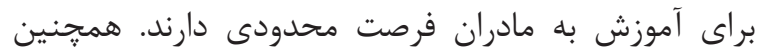

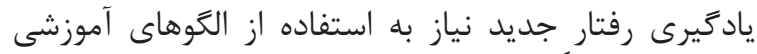

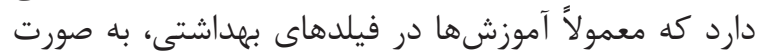

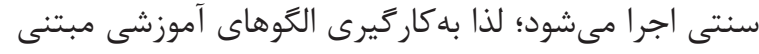

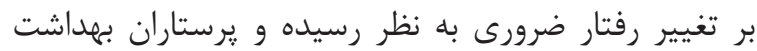

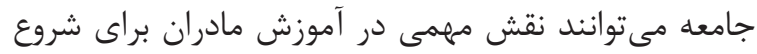

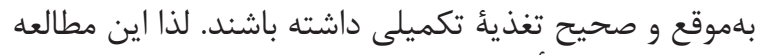

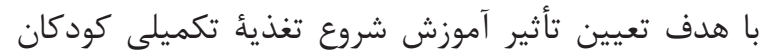

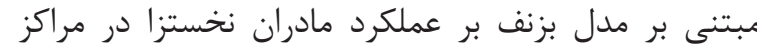

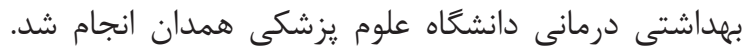

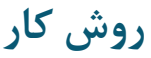

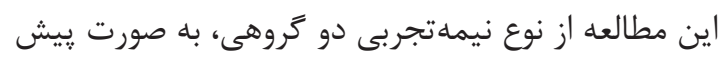

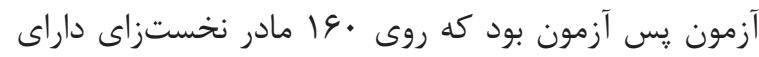

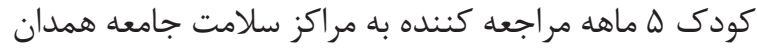

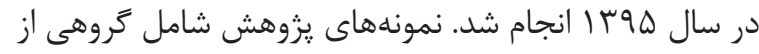

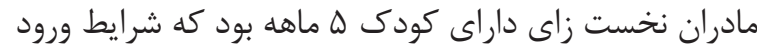

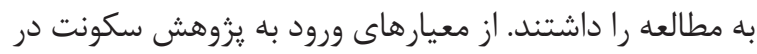

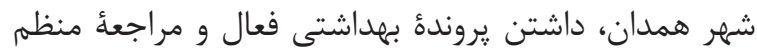

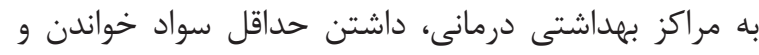

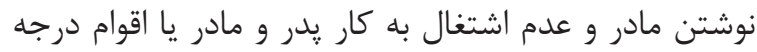

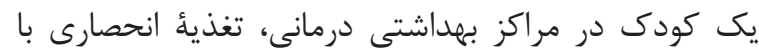

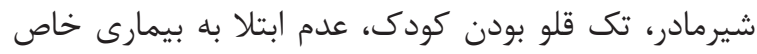

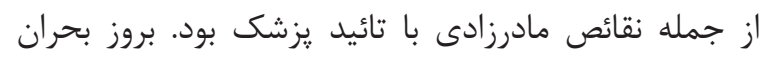

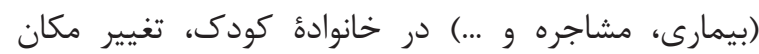

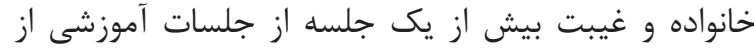
جمله معيارهاى خروج از مطالعه بود.

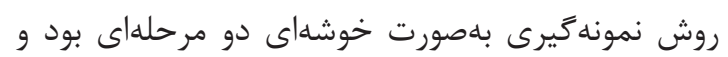

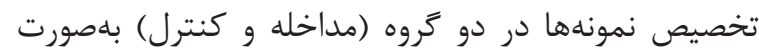

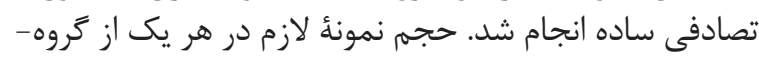

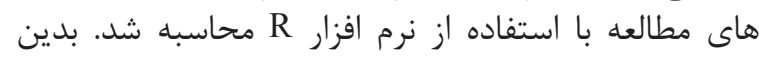

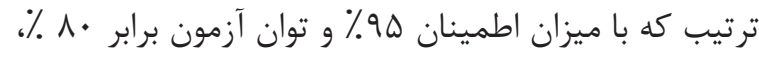
همجنين اختلاف مورد انتظار

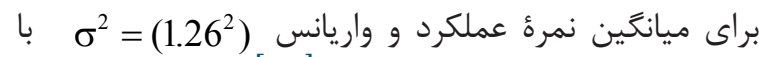

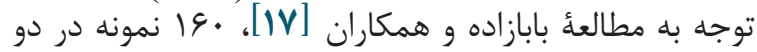
كروه تعيين شد.

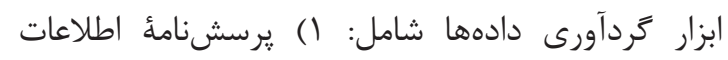

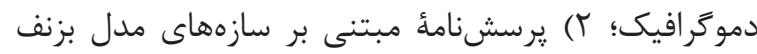

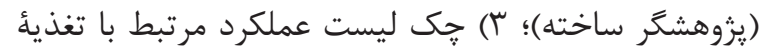
تكميلى (يزوهشكر ساخته) بود.

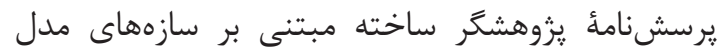

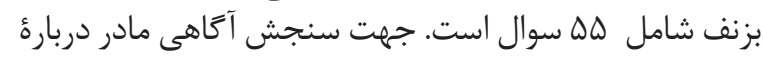

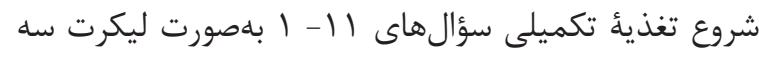

\section{Hubley}

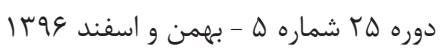

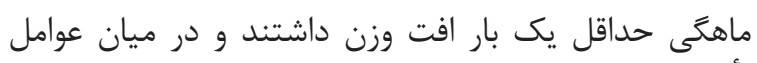

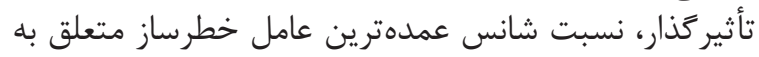

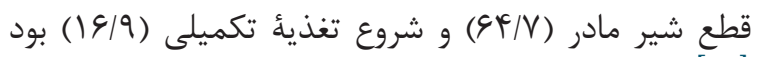

زمانى كه تغذئُ كودى از شير مادر به غذاى سفره تغيير

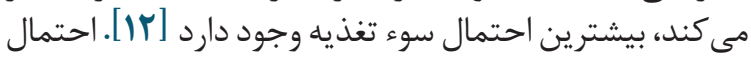

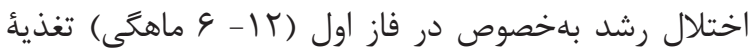
تكميلى، بيشتر است. در مطالعات متعدد برد بر الهميت فراز فرايند تغذئُ تكميلى تاكيد شده [سأ

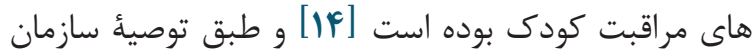

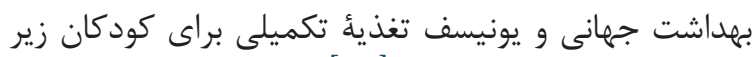

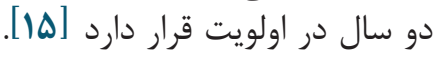

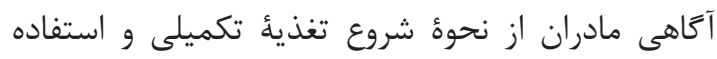

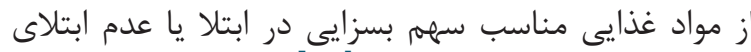

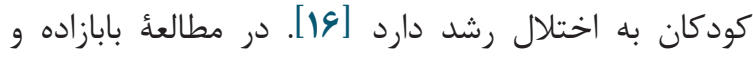

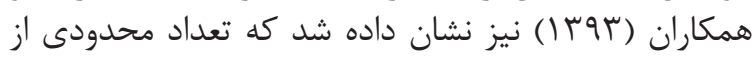

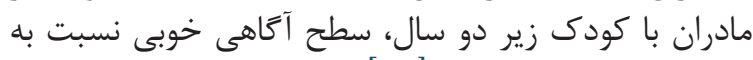

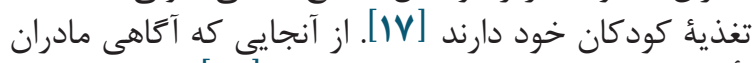

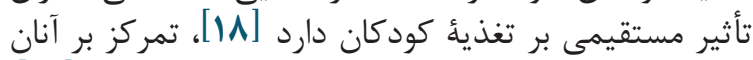

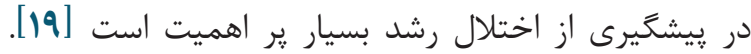

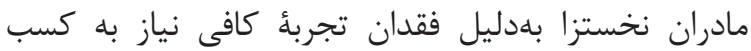

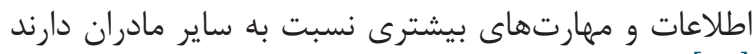

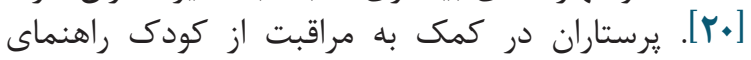

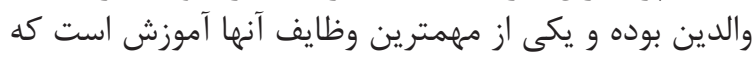

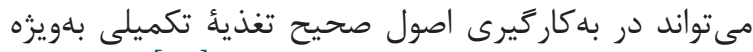

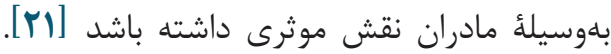

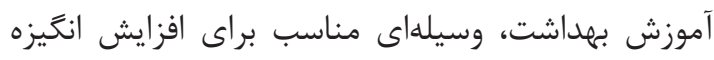

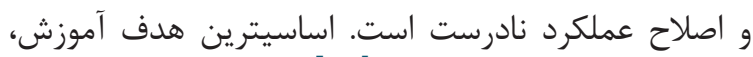

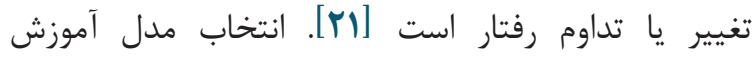

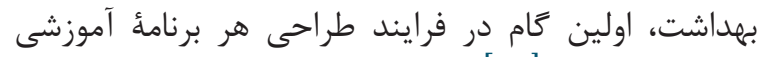

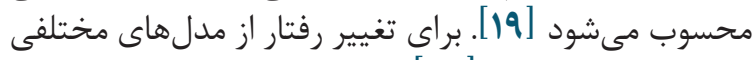

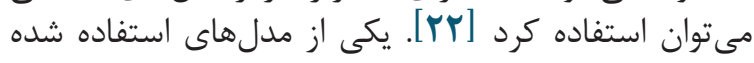

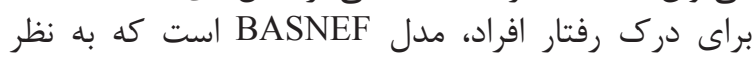

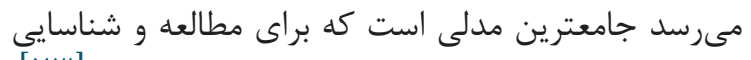

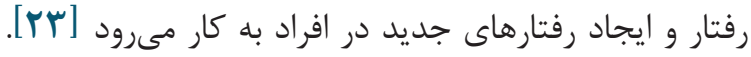

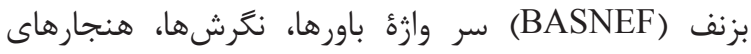

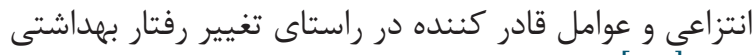

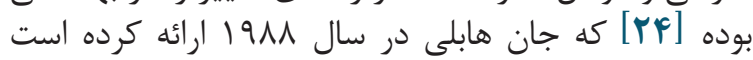

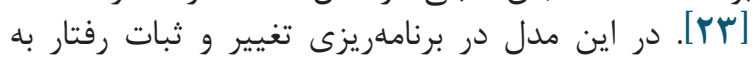

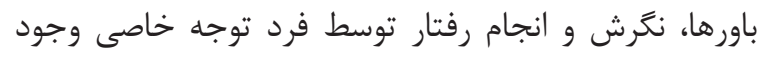

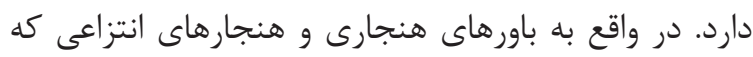

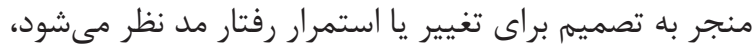

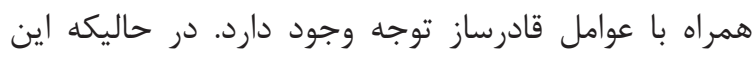

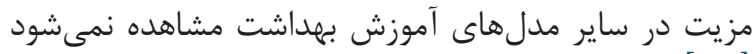

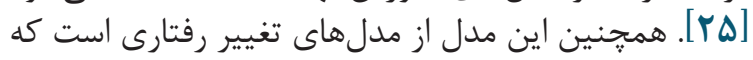
بلهصورت بالقوه براى برنامههاى آموزش آماى تغذيه مناسب است است [rq] علىرغم اهميت نحوه شروع تغذية تكميلى، در عمل 
شامل يرسشنامهٔ اطلاعات دموكرافيك، يرسشنامةٔ مبتنى بر

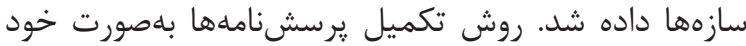

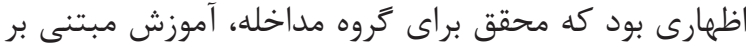

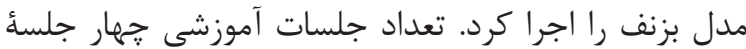

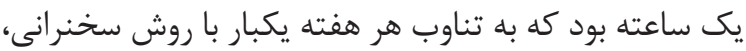

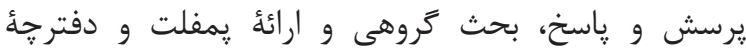

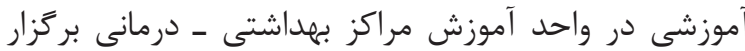

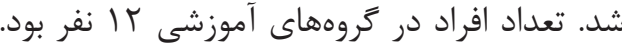
كروه كنترل، بلهورت روتين به مراكز بهداشتى درمانى

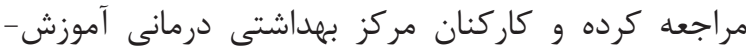

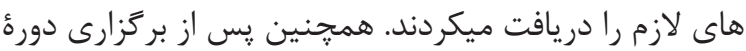

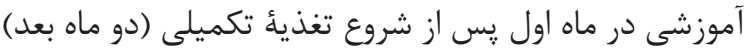

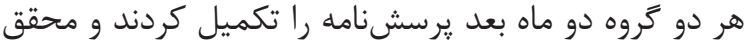

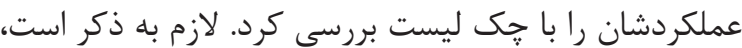

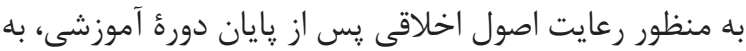

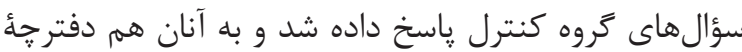
آموزشى داده شد.

براى تجزيه و تحليل دادهها از نرم افزار SPSS نسخهُ

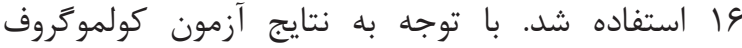

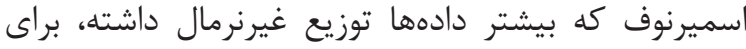

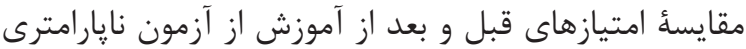

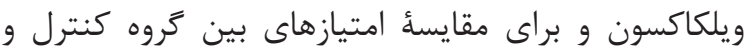

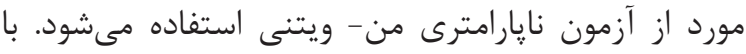

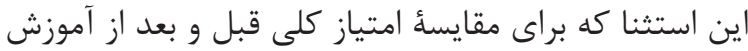

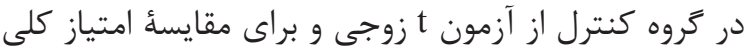

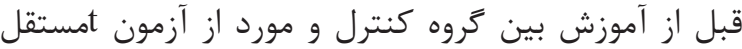
ستفاده شد.

\section{يافتهها}

ميانگين سنى مادران در دو گروه مداخله و كنترل به ترتيب TV ميانگين سن يدران در گروه مداخله كروه كنترل ميانكن

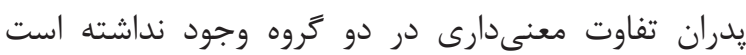

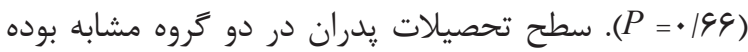

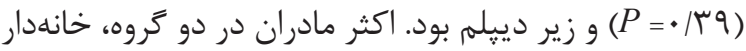

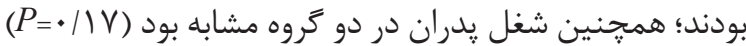

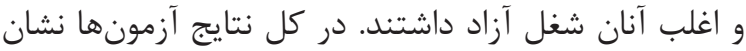
داد كه هيج گونه اختلاف معنادارى از لحاظ إن سن، سطح

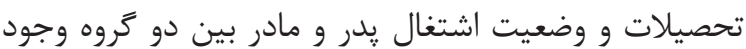

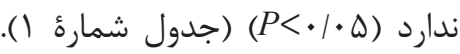
ميانگين نمرهكلى و نمرة هر يك از ساختارهاى مدل بزنف

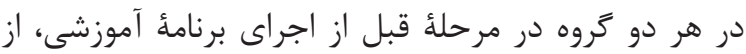

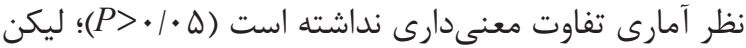

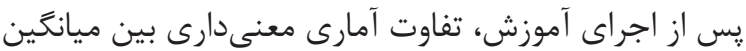

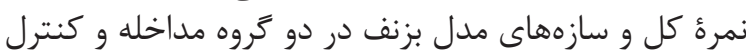

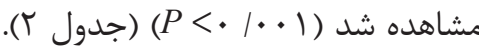

كزينهاى (صحيح، غلط، نمى نقانم) طراحى شده است. به

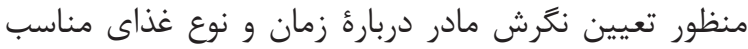

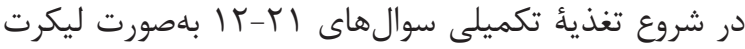

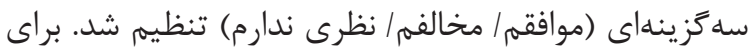

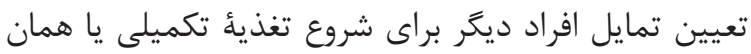

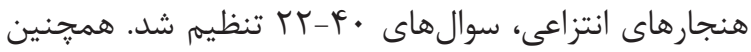

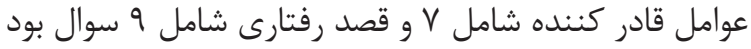

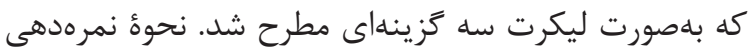

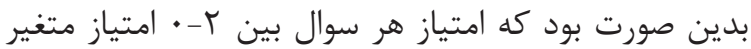

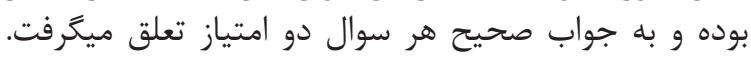

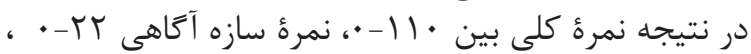

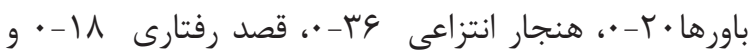

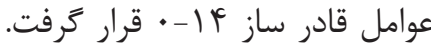

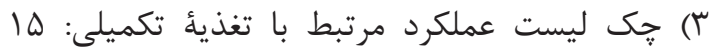

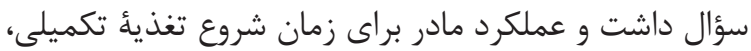

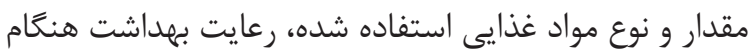

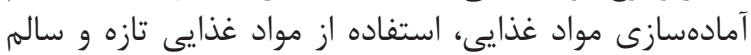

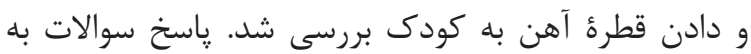

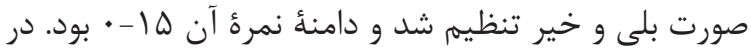

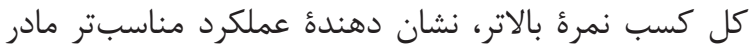

در اين مطالعه براى بررسى روايى محتوايى به شكل كمى،

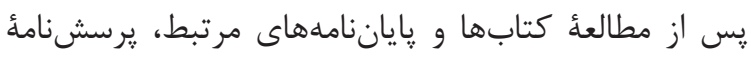

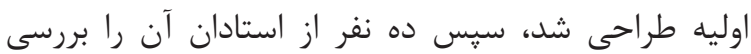

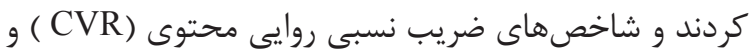

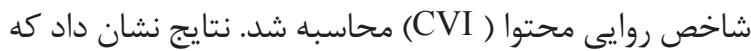
براى تك تك سؤال هاى يرسش مجنامه، مقادير CVI و

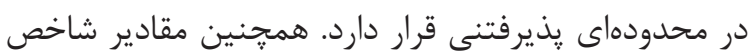

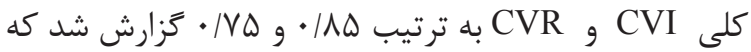

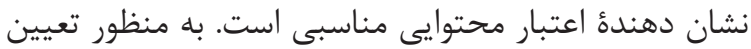

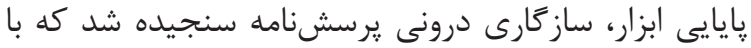

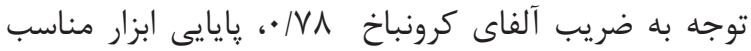
تشخيص داده شد.

روش كار بدين صورت بود كه يس از انجام بررسى

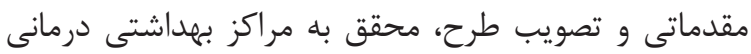

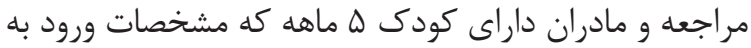

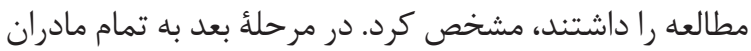

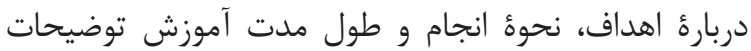

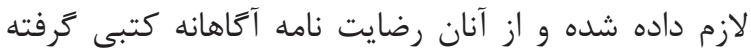

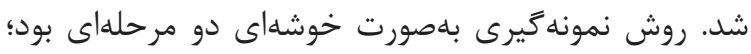

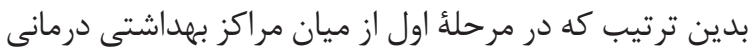

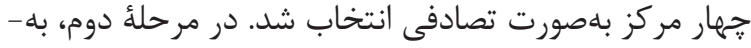

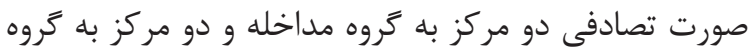

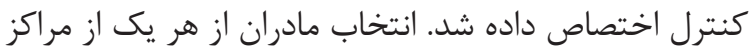

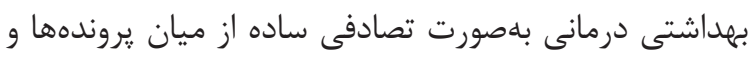

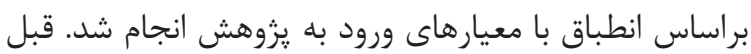

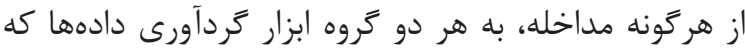


جدول 1. مقايسُٔ مشخصات دموكر افيك تروههاى مداخله و كنترل

\begin{tabular}{|c|c|c|c|c|}
\hline \multirow{2}{*}{ نتايج آزمون آمارى } & \multicolumn{2}{|c|}{ فراوانى(\%) | } & \multirow{2}{*}{\multicolumn{2}{|c|}{ متغير }} \\
\hline & كروه كنترل & كروه مداخله & & \\
\hline \multirow{3}{*}{$\begin{array}{c}\text { Chir- Test } \\
X^{r}=1 / \Psi \cdot V \\
\text { P-Value }=\cdot / V 9 r\end{array}$} & $r q(\% \varphi \Delta / \cdot \cdot)$ & $\mu r(\% \Delta r / V \Delta)$ & زير دييلم & \multirow{3}{*}{ سطح تحصيلات مادر } \\
\hline & ऍ (\% & $r \omega(\% \Gamma / / T \omega)$ & ديبلم & \\
\hline & $1 \Gamma(\% \mid g / r \Delta)$ & $\mid r(\% \mid \Delta)$ & ليسانس و بالاتر از ليسانس & \\
\hline \multirow{2}{*}{$\begin{array}{c}\text { Chir- Test } \\
X^{r}=\cdot / r \cdot \varphi \\
\text { P-Value }=1 / \cdot \cdots\end{array}$} & $r(\% / \vee \Delta)$ & $r(\% / \Delta \cdot)$ & شاغل & \multirow{4}{*}{ وضعيت اشتغال مادر } \\
\hline & $V \vee(\% ৭ 9 / r \Delta)$ & $\vee \wedge(\% ৭ \vee / \Delta \cdot)$ & 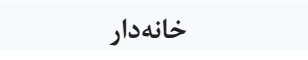 & \\
\hline $\begin{array}{c}\text { Independent } T \text { Test } \\
t=\cdot / r \cdot \Lambda\end{array}$ & ميانكين \pm انحراف معيار & ميانكين \pm انحراف معيار & \multirow[t]{2}{*}{ سن مادر (سال) } & \\
\hline $\mathrm{P}-$ Value $=\cdot / \mathrm{V} \Delta \Lambda$ & $r V / \Lambda D_{ \pm} \Delta / r \Lambda$ & $r N / I T \pm \Delta / q V$ & & \\
\hline
\end{tabular}

جدول شماره r. مقايسهُ ميانگين نمرات شروع تغذيئ تكميلى در دو كروه مداخله و كنترل قبل و بعد از آموزش

\begin{tabular}{|c|c|c|c|c|c|}
\hline \multicolumn{2}{|c|}{ 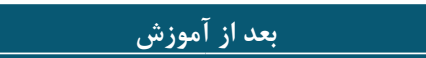 } & \multicolumn{2}{|c|}{ ق قبل از آموزش } & \multirow{3}{*}{ محدوده نمره } & \multirow{3}{*}{ سازهها } \\
\hline تروه كنترل & تروه مداخله & تروه كنترل & تروه مداخله & & \\
\hline Mean \pm SD & Mean \pm SD & Mean \pm SD & Mean \pm SD & & \\
\hline $19 / 4 \cdot \pm r / 4 r$ & $r / 9 \Lambda_{ \pm} \cdot / 11$ & $19 / r \Lambda_{ \pm} r / V \varphi$ & $19 / 94 \pm r / 11$ & $\cdot-t r$ & \multirow{2}{*}{ آنَاهى } \\
\hline$Z=-\Lambda / v \Delta \Lambda$ & $P=\cdot / \cdot$ & \multicolumn{2}{|c|}{$\mathrm{Z}=-1 /$ $q \vee \quad P$} & & \\
\hline $1 Q / q V \pm r / r \Lambda$ & $\mid V / 91 \pm \cdot / 11$ & $\left|\varepsilon / r \omega_{ \pm} r /\right| r$ & $19 / 9 \mathrm{~V} \pm 1 / \Delta \Lambda$ & $\cdot-r \cdot$ & \multirow{2}{*}{ 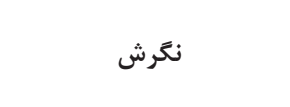 } \\
\hline $\mathrm{Z}=-\mathrm{V} / 9 \mid \mathrm{V}$ & $P=\cdot / \cdots$ & \multicolumn{2}{|c|}{$\mathrm{Z}=-\cdot / \uparrow q \varphi \quad P$} & & \\
\hline$r / / r \Delta \pm r / \Delta \Lambda$ & $r \Delta / 91 \pm \cdot / r \varphi$ & $r M / l \cdot \pm \Delta / F V$ & $r \cdot / V r \pm \Delta / V r$ & $\cdot-r_{4}$ & \multirow{2}{*}{ هنجارهاى انتزاعى } \\
\hline$Z=-\Lambda / \Delta \Lambda \Lambda$ & $P=\cdot / \cdots$ & \multicolumn{2}{|c|}{$\mathrm{Z}=-\cdot / r \mu \Delta \quad P$} & & \\
\hline $1 r / 99 \pm r / 9 r$ & $1 \Delta / \Lambda T_{ \pm} \cdot / \Delta 9$ & $\mid r / \Lambda \cdot \pm r / 9 r$ & \multirow{2}{*}{$|r / 9| \pm T / F r$} & $\cdot-14$ & \multirow{2}{*}{ عوامل قادركننده } \\
\hline$Z=-\Lambda / \varphi \Lambda$ & $P=\cdot / \cdot$ & $\mathrm{Z}=-\cdot /$ NT $\quad P=$ & & & \\
\hline $\mid r / M \pm \pm r / r$. & $\mid \psi / \cdot \cdot \pm \cdot \cdot \cdot$ & $\mid r / T \Lambda \pm T / T V$ & $\begin{array}{l}Z=-V / G F T \\
P=\cdot / \cdots\end{array}$ & $\cdot-11$ & \multirow{2}{*}{ قصد رفتارى } \\
\hline$Z=-V / G F T$ & $P=\cdot / \cdots$ & \multicolumn{2}{|c|}{$\mathrm{Z}=-\cdot / V \vee \wedge \quad P$} & & \\
\hline $91 / \Delta V \pm 9 / \wedge \Lambda$ & $1 \cdot \Delta / V I \pm \cdot / 9 f$ & $91 / A T \pm 1 \cdot / \Delta T$ & \multirow{2}{*}{$\begin{array}{r}q r / \cdot \Delta \pm 1 \cdot / \Lambda r \\
r_{\Delta} \quad P\end{array}$} & $\cdot-11$ & \multirow{2}{*}{ نمرأكلى } \\
\hline$Z=-1 \cdot / 4 r \Delta$ & $P=\cdot / \cdots$ & & & & \\
\hline $11 / 91 \pm 1 / 19$ & $\mid \& / \wedge \Delta \pm \cdot / 9 \Delta$ & \multirow{2}{*}{\multicolumn{2}{|c|}{--}} & $\cdot-10$ & \multirow{2}{*}{ نمرهٔ عملكرد شروع تغذيئ } \\
\hline$Z=-9 / r \Lambda \Lambda$ & $P=\cdot / \cdots$ & & & & \\
\hline
\end{tabular}

جدول شماره ب. مقايسة ميانغين نمرات شروع تغذية تكميلى قبل و بعد از آموزش در هر يك از دو كَروه مداخله و كنترل

\begin{tabular}{|c|c|c|c|c|c|c|}
\hline & كنترل & & & مداخله & & \\
\hline آزمون آمارى & $\begin{array}{c}\text { بعد } \\
\text { Mean }_{ \pm} \text {SD }\end{array}$ & $\underset{\text { Mean }}{\text { قبل SD }}$ & آزمون آمارى & $\begin{array}{c}\text { بعean } \\
\text { Mead }\end{array}$ & $\begin{array}{c}\text { قبل } \\
\text { Mean } \pm \text { SD }\end{array}$ & سازهها \\
\hline $\begin{array}{l}\mathrm{Z}=-\cdot / \pi \mu \mid \\
P=\cdot|\Lambda| \Lambda\end{array}$ & $19 / 4 \cdot \pm r / F r$ & $19 / r \wedge \pm r / V \varphi$ & $\begin{array}{l}\mathrm{Z}=-9 / \Gamma \cdot \varphi \\
P=\cdot / \cdot \cdots\end{array}$ & $r \backslash / 9 \Lambda_{ \pm} \cdot / 11$ & $\left|9 / 9 \varepsilon_{ \pm} \Gamma / 1\right|$ & آكاهى \\
\hline $\begin{array}{l}\mathrm{Z}=-1 / 09 \\
P=\cdot 1.9 \vee\end{array}$ & $\mid \omega / q \vee \pm r / \Upsilon \Lambda$ & $19 / \pi \Delta \pm r / 1 r$ & $\begin{array}{l}\mathrm{Z}=-\Delta / V \Delta T \\
P=\cdot 1 \cdot \cdots\end{array}$ & $|V / 91 \pm \cdot / 1|$ & $1919 \mathrm{~V} \pm 1 / 01$ & 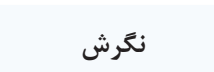 \\
\hline $\begin{array}{l}Z=-\cdot / V \wedge \\
P=\cdot / 9 \mathrm{VV}\end{array}$ & $r 1 / r \Delta \pm \Psi / \Delta \Lambda$ & $r / / 1 \cdot \pm \Delta / \notin v$ & $\begin{array}{l}\mathrm{Z}=-9 / T F \varphi \\
P=\cdot / \cdot \ldots\end{array}$ & $r \Delta / 91 \pm \cdot r \varphi$ & $r \cdot / V r \pm \Delta / V r$ & هنجارهاى انتزاعى \\
\hline $\begin{array}{l}\mathrm{Z}=-1 / \Delta \mathrm{V} \\
P=\cdot / 1 \mathrm{r}^{\prime}\end{array}$ & $\mid r / 99 \pm r / 9 r$ & $\mid r / \Lambda \cdot \pm r / 9 r$ & $\begin{array}{l}\mathrm{Z}=-9 / 190 \\
P=\cdot 1 \cdot \ldots\end{array}$ & $\mid \Delta / \Lambda r_{ \pm} \cdot / \Delta q$ & $\mid r / q 1 \pm r / F r$ & عوامل قادر كننده \\
\hline $\begin{array}{l}\mathrm{Z}=-\cdot / 9 \mathrm{~T} \\
P=\cdot / \mathrm{VVT}\end{array}$ & $|r /| \Lambda \pm r / r$. & $\mid r / T \Lambda \pm r / T V$ & $\begin{array}{l}\mathrm{Z}=-\Delta / 9 \wedge \Delta \\
P=\cdot / \cdot \cdots\end{array}$ & $|\psi| \cdot{ }_{ \pm} \cdot / \cdot$ & $|r / V V \pm| / F \mid$ & قصد رفتارى \\
\hline $\begin{array}{c}\mathrm{t}=\cdot / \mathcal{F F} \\
P=\cdot / \& V K\end{array}$ & $9 / / \Delta V \pm 9 / \wedge \Lambda$ & $91 / A r \pm 1 \cdot / \Delta r$ & $\begin{array}{c}\mathrm{Z}=-\mathrm{V} / 1 \mathrm{~V} \mathrm{~V} \\
P=\cdot / \cdots\end{array}$ & $1 \cdot \Delta / V I \pm \cdot / 9 F$ & $q r / \cdot \Delta \pm 1 \cdot / \Lambda r$ & نمرء كلى \\
\hline
\end{tabular}

سن شروع تغذيئ تكميلى در گروه مداخله

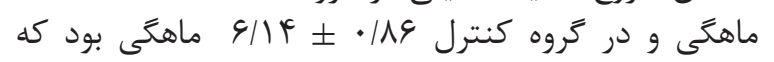

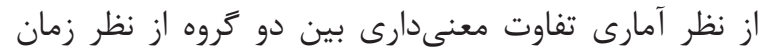

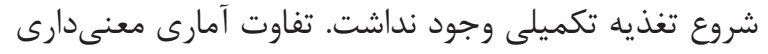

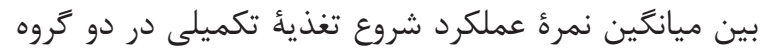

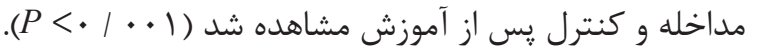

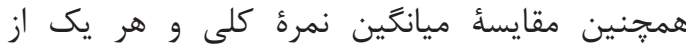

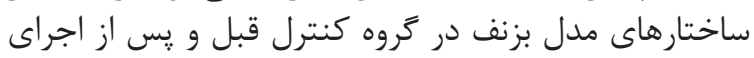

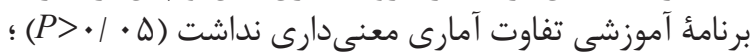

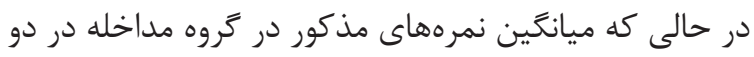

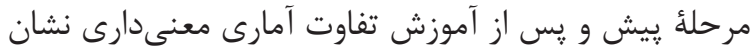

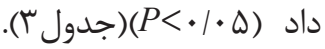


بلاويزه مادران و مادربزرَّها در عملكرد تغذئُ تكميلى مادر

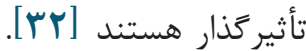

مقايسٔ عوامل قادركنندة شروع تغذئه تكميلى مادران

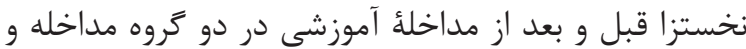

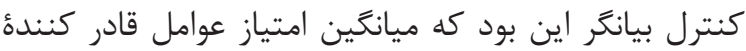

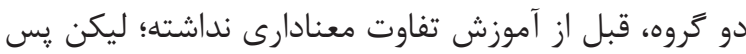

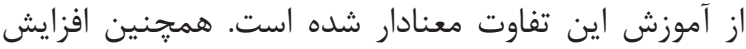

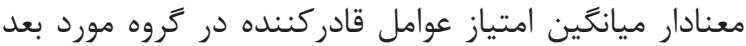

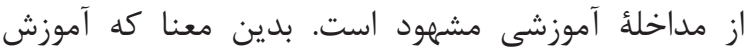

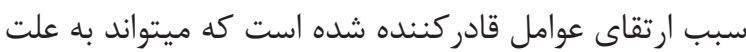

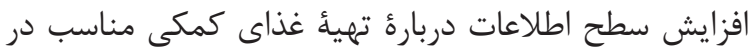

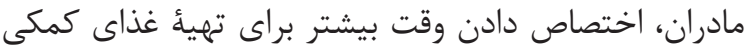

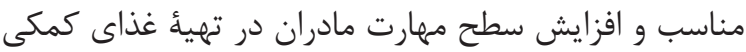

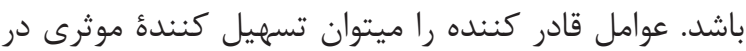

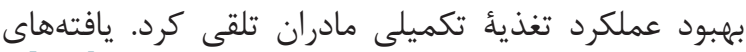

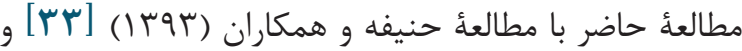

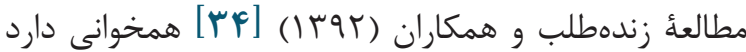

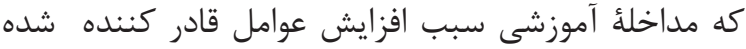

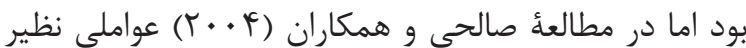

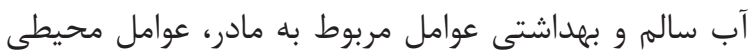

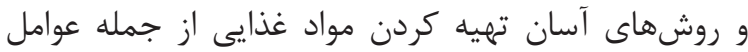

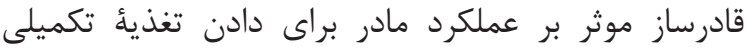

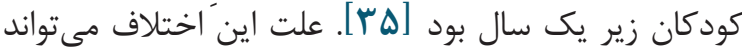

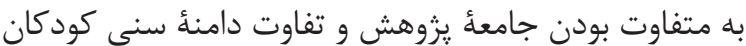

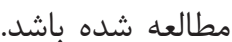

نتايج اين مطالعه نشان داد كه ميانكين امتياز قصد رفتارى

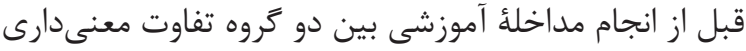

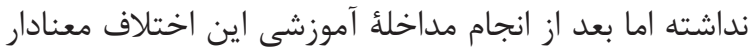

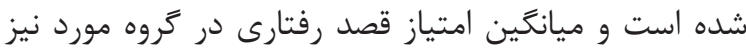

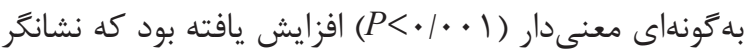

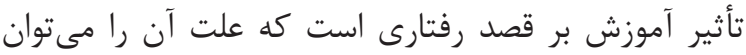

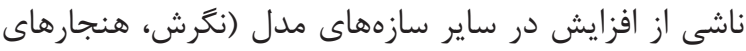

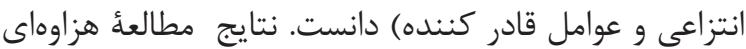

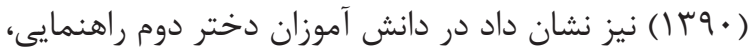

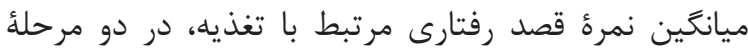

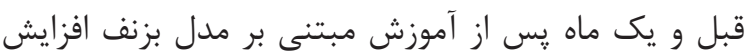

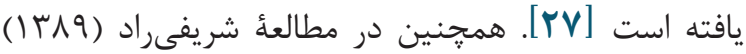

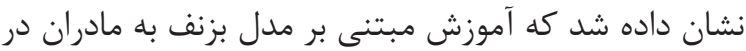

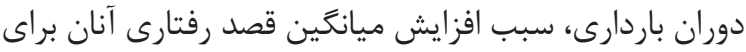

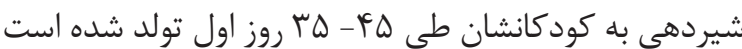

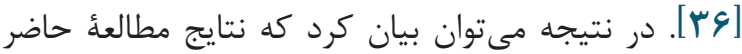

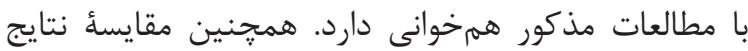

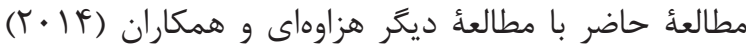

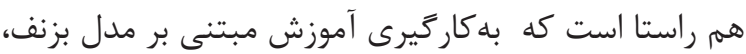

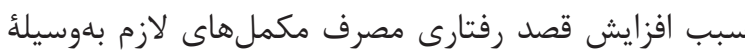

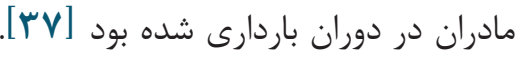

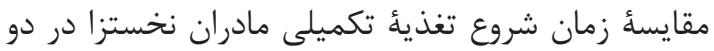

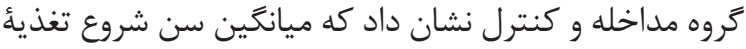

دوره T شماره ه - بهمن و اسفند وqجا
بحث

هدف از انجام اين مطالعه تعيين تأثير آموزش مبتنى بردي

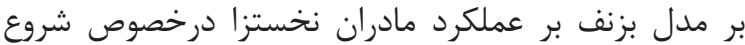

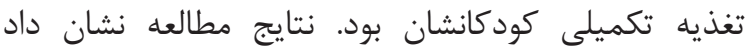

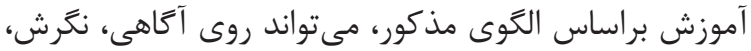

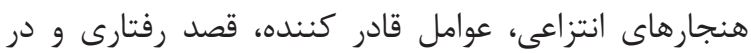

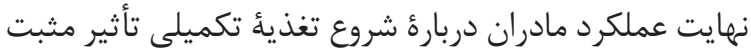

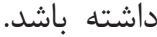

مقايسٔ آكاهى نحوه شروع تغذيئ تكميلى كودكان مادران

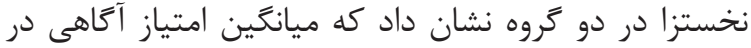

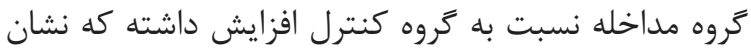

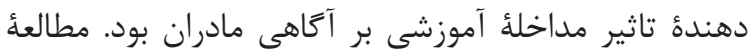

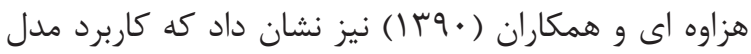

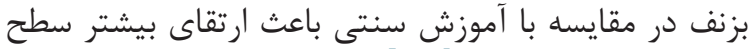

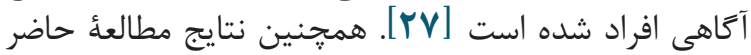

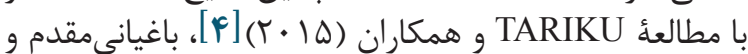

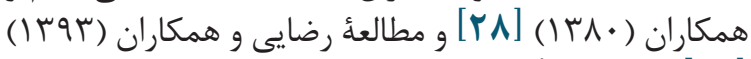

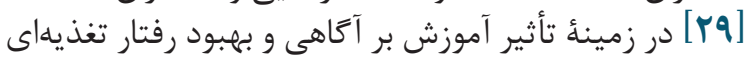
نمونهاى مطالعهشان همخوانى داشت آنت

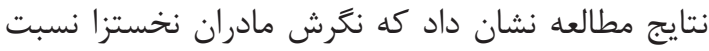

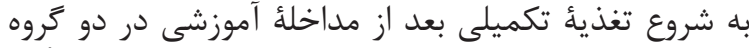

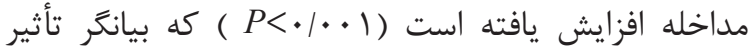

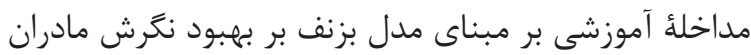

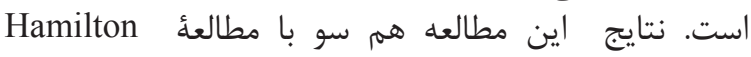

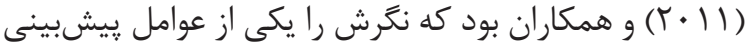

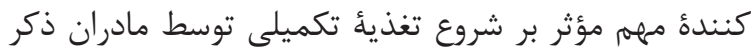

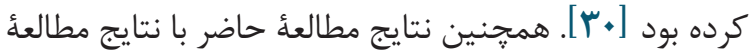

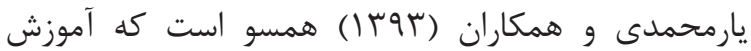

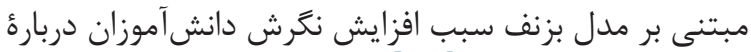

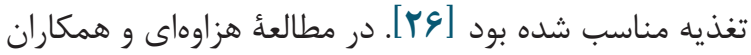

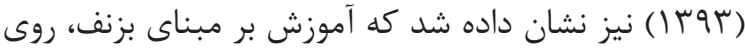

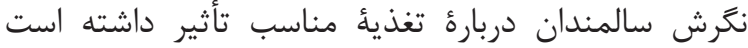

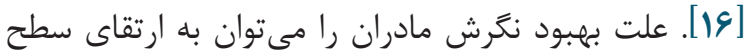

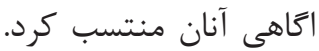

مقايسٔ هنجارهاى انتزاعى شروع تغذئ تكميلى مادران

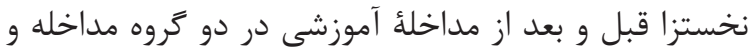

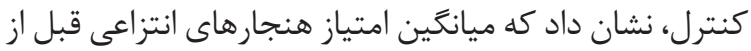

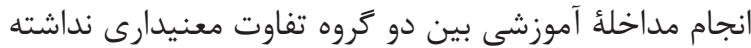
إما

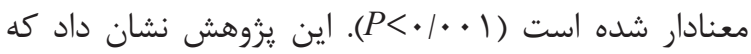

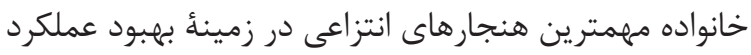

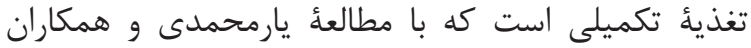

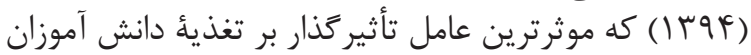

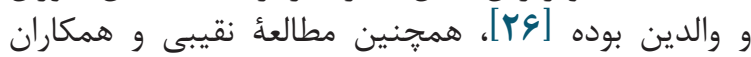

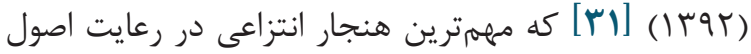

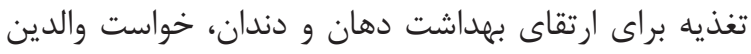

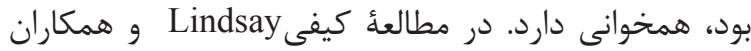

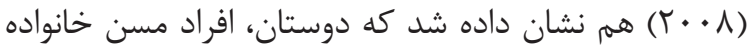




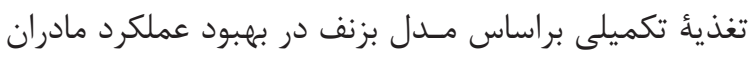

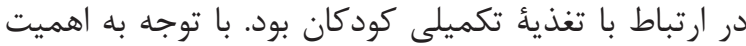

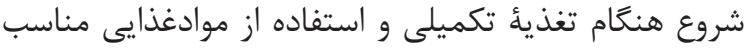

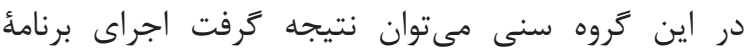

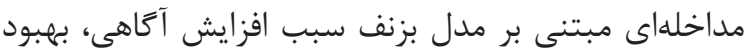

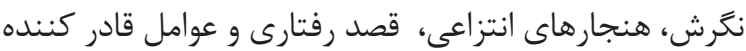

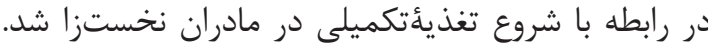

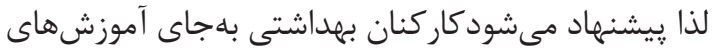

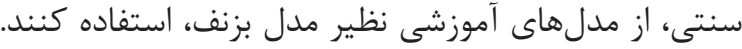

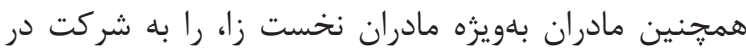

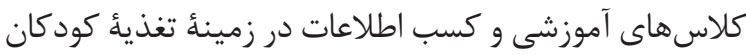

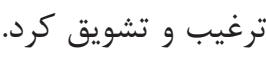

\section{سياسگَزارى}

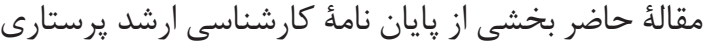

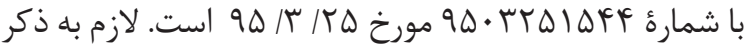

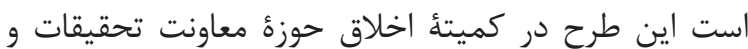

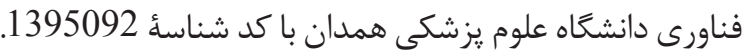

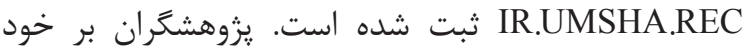

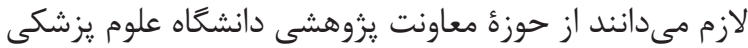

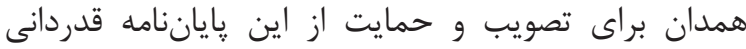

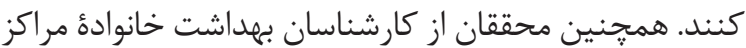

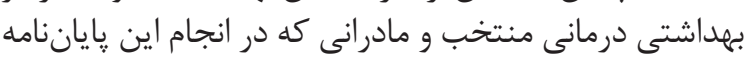

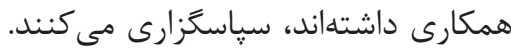

$$
\text { تعارض در منافع }
$$

بين نويسندًان مطالعه تضاد منافعى وجود ندارد.
تكميلى در گروه مداخله و كنترل تفاوت معنى دارى ندار ندارد

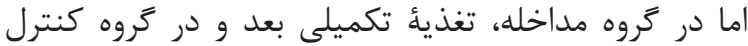

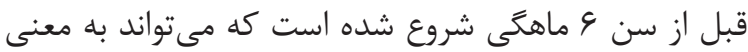

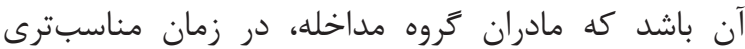

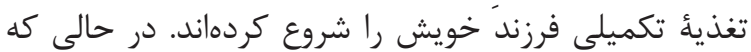

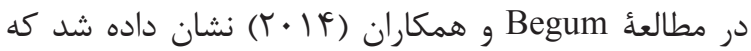

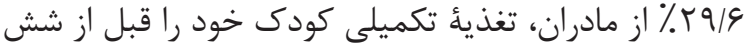

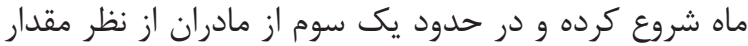

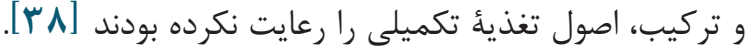

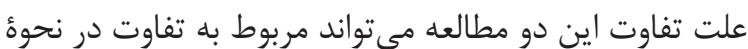
آموزش مادران مطالعه شده باشد.

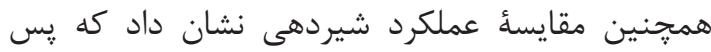

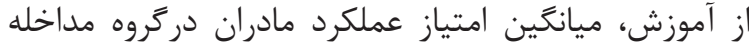

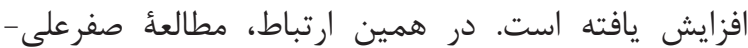

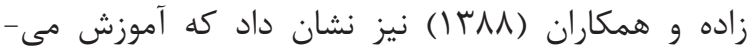

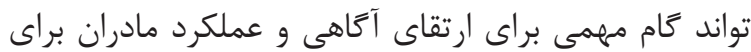

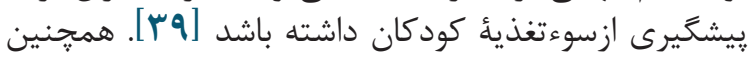

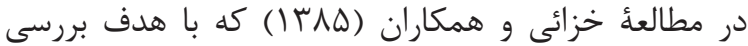

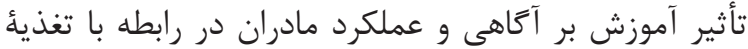

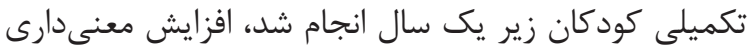

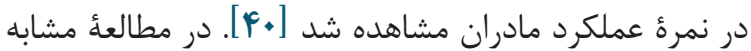

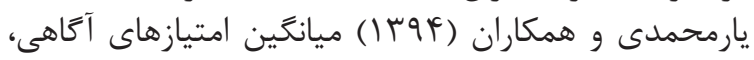

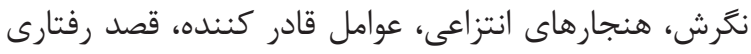

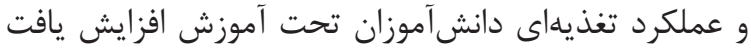

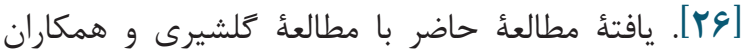

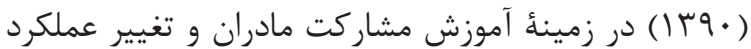

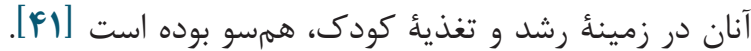

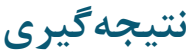

نتايج مطالعئ حاضر بيانكر اهميت طراحى برنامهٔ آموزش

\section{References}

1. Lange C, Visalli M, Jacob S, Chabanet C, Schlich $\mathrm{P}$, Nicklaus S. Maternal feeding practices during the first year and their impact on infants' acceptance of complementary food. Food Qual Prefer. 2013;29(2):89-98. https://doi.org/10.1016/j. foodqual.2013.03.005

2. WHO. Guiding principles for feeding non-breastfed children 6-24 months of age. 2005:1-40.

3. Imdad A, Yakoob MY, Bhutta ZA. Impact of maternal education about complementary feeding and provision of complementary foods on child growth in developing countries. BMC Public Health. 2011;11(3):S25. https://doi.org/10.1186/1471-2458-11-S3-S25 PMid:21501443 PMCid:PMC3231899

4. Tariku B, Whiting SJ, Mulualem D, Singh P. Application of the Health Belief Model to Teach Complementary Feeding Messages in Ethiopia. Ecol Food Nutr. 2015;54(5):572-82. https://doi.org/10.1080/03670244.2015.1049344
PMid:26075935

5. Shidfar F, Montazer M, Azizi H, Darvishian M, Jahangiri N. The relation between age of introduction of complementary feeding and physical growth of infants under 2 years of age in west of Tehran. Razi Journal of Medical Sciences. 2008;14(57):121-31.

6. Salarkia N, Amini M, Eslami M, Dadkhah M, Zoghi T, Haidari H, et al. Mothers' views and beliefs about the role of complementary feeding in children under the age of two in Damavand: a qualitative study. J Arak Univ Med Sci. 2010;13(2):63-74.

7. Saleem AF, Mahmud S, Baig-Ansari N, Zaidi AK. Impact of maternal education about complementary feeding on their infants' nutritional outcomes in low-and middle-income households: a community-based randomized interventional study in Karachi, Pakistan. J Health Popul Nutr. 2014;32(4):623-33. PMid:25895196 PMCid:P- 


\section{MC4438693}

8. WHO. Essential nutrition actions: improving maternal, newborn, infant and young child health and nutrition: World Health Organization; 2013.

9. Black RE, Cousens S, Johnson HL, Lawn JE, Rudan I, Bassani DG, et al. Global, regional, and national causes of child mortality in 2008: a systematic analysis. Lancet. 2010;375(9730):1969-87. https://doi.org/10.1016/S0140-6736(10)60549-1

10. Shi L, Zhang J, Wang Y, Caulfield LE, Guyer B. Effectiveness of an educational intervention on complementary feeding practices and growth in rural China: a cluster randomised controlled trial. Public Health Nutr. 2010;13(04):556-65. https://doi.org/10.1017/S1368980009991364 PMid:19706219

11. Kholdi N, Ramezankhani A, Zayeri F, Jafari F, Davati A. Weight Faltering and its Major Immediate Risk Factors up to 2 Years of Age in Children Attending Health Care Centers, East of Tehran. Pajoohandeh. 2010;15(3):104-10.

12. Khanal V, Sauer K, Zhao Y. Determinants of complementary feeding practices among Nepalese children aged 6-23 months: findings from demographic and health survey 2011. BMC Pediatr. 2013;13(1):131-8. https://doi. org/10.1186/1471-2431-13-131 PMid:23981670 PMCid:PMC3766108

13. Salarkia N, Amini M, Abdollahi M, Eshrati B, Neyestani RT. Mothers' Views on Food Security and Complementary Feeding: A Qualitative Study in Urban Iran. Nutr Food Sci Res. 2014;1(2):35-42.

14. Abdollahi F, Yazdani-Charati J, Rohani S. Study of Complementary Feeding Practices and Some Related Factors among Mothers Attending Primary Health Centers in Sari, Iran, in 2013. Iran J Health Sci. 2014;2(3):43-8. https://doi. org/10.18869/acadpub.jhs.2.3.43

15. WHO. Nutrition - Child and Adolescent Health and Development,. Available from: http://wwwwhoint/child-adolescent-health/ NUTRITION/ complementary.htm Accessed July 13. 2007.

16. Hazavehi MM, Taheri M, Moeini B, Roshanaei $\mathrm{G}$. The effect of educational program on mother's child (6 to 9 month age) for reducing growth failure in Hamadan: applying Health Belief Model (HBM). Jundishapur Journal of Health Sciences. 2013;5(2):129-39.

17. Babazade T, Moradi F, Zibaee N. Impact of educational intervention on mothers Empowerment about proper nutrition in 0-2 years infants covered by Homes of Health district 18 of Tehran. J Health Educat Health Promotion. 2014;2(3):24250.

18. Baghdari N, Bahrami Morghaki A, Norozi A, Sadeghi Sahebzad E, Mazlom SR, Mohajeri A.
The Effect of Mother's Training via Newsletter and Group Discussion on The Energy Intake of Preschool Children. Iran J Nurs Midwifery Res. 2014;2(3):180-7.

19. Kashfi SM, Jeyhouni AK. Mothers' educational program based on the precede model on the prevention of growth retardation in 6 to 12-month old children in the health centers of Shiraz city, Fars Province, Iran: An Interventional Study. J Sch Public Health Inst Public Health Res. 2012;9(3):55-63.

20. Holambe V, Thakur N. Factors affecting late introduction of complementary food: A kaplan meier analysis. Indian J Community Med. 2014;5(1):140-3.

21. Smeltzer J, Neiger B, Mckenzie A. Planning, Implementing and Evaluating Health Promotion Programs. USA: Pearson Benjamin cummings; 2005.

22. Glanz K, Rimer BK, Viswanath K. Health behavior and health education: theory, research, and practice. New Jersey: John Wiley \& Sons; 2008.

23. Hubley J. Understanding behaviour: the key to successful health education. Trop Doct. 1988;18(3):134-8. https://doi.org/10.1177/0049 47558801800316 PMid: $\underline{3406993}$

24. Saffari M, Shojaeizadeh D, Ghofranipour F, Heydarnia A, Pakpour A. Health Education \& Promotion-Theories, Models \& Methods. Tehran: Sobhan Publication; 2009:55-7.

25. Baghianimoghadam MH, Rahaee Z, Morowatisharifabad MA, Sharifirad G, Andishmand A, Azadbakht L. Effects of education on self-monitoring of blood pressure based on BASNEF model in hypertensive patients. J Res Med Sci. 2010;15(2):70-7. PMid:21526062 PMCid:PMC3082790

26. Yarmohammadi S, Mahmoodi M, Jazayeri SA, Chamari M. The effect of an educational program based on the BASNEF model on the nutritional behavior of guidance school female pupils. J Sch Public Health Inst Public Health Res. 2015;13(2):55-68.

27. Hazavehei SMM, Pirzadeh A, Entezari MH, Hasanzadeh A. The effect of educational program based on BASNEF model on the nutritional behavior of students. Zahedan J Res Med Sci. 2011;13(1):23-9.

28. Baghianimoghadam M. The study of BASNEF Model effectiveness in controlling Yazd diabetic patients. Tehran: Tarbiatmodares University; 2001.

29. Rezaei OM, Moodi M, Moazam N. Analyzing the level of knowledge and attitude of the mothers referring the urban health centers of Birjand about nutritional behaviors. J Educ Health Promot. 2014;3(1):37. PMid:25013830 PMCid:P- 


\section{MC4089104}

30. Hamilton K, Daniels L, White KM, Murray N, Walsh A. Predicting mothers' decisions to introduce complementary feeding at 6 months. An investigation using an extended theory of planned behaviour. Appetite. 2011;56(3):67481. https://doi.org/10.1016/j.appet.2011.02.002 PMid:2131641

31. Naghibi SA, Yazdani Cherati J, Khujeh Z, Shah Hosseini M. Factors Influencing Oral Health Behavior According to BASNEF Model. J Mazandaran Univ Med Sci. 2013;23(99):76-83.

32. Lindsay AC, Machado MT, Sussner KM, Hardwick CK, Peterson KE. Infant-feeding practices and beliefs about complementary feeding among low-income Brazilian mothers: a qualitative study. Food Nutr Bull. 2008;29(1):15-24. https://doi.org/10.1177/156482650802900102 PMid:18510201

33. Hanifeh A, Majlesi F, Tol A, Mahmodi Majdabadi M, Nasiri A. Assessing the effect of educational intervention based on BAZNEF model on physical activity of female university students. Razi Journal of Medical Sciences. 2014;21(122):9-19.

34. Zendehtalab H, Vaghei S, Emamimoghadam Z. Effect of intervention based on BASNEF model on quality of life in patients with type 2 diabetes. Evidence Based Care. 2013;3(1):7-16. http://dx. doi.org/10.22038/ebcj.2013.565

35. Salehi M, Kimiagar SM, Shahbazi M, Mehrabi Y, Kolahi A. Assessing the impact of nutrition education on growth indices of Iranian nomadic children: an application of a modified beliefs, attitudes, subjective-norms and enabling-factors model. Br J Nutr. 2004;91(5):779-87. https://doi. org/10.1079/BJN20041099 PMid: $\underline{15137930}$

$$
\text { 1M 1. تأثير آموزش مبتنى بر مدل بزنف بر ... }
$$

36. Sharifirad G, Golshiri P, Shahnazi H, Barati M, Hassanzadeh A. The impact of educational program based on BASNEF model on breastfeeding behavior of pregnant mothers in Arak. Arak Medical University Journal. 2010;13(1):63-70.

37. Hazavehei M, Etesamifar T, Moeini B, Roshanaei G, Mahboubi M. Education based on BASNEF model; an affective education on regular use of nutritional supplements during pregnancy. J Biol Todays World. 2014;3:174-9. https://doi. org/10.15412/J.JBTW.01030803

38. Begum T, Hoque SA, Islam MR, Katoon S, Shah AR. Infant feeding practice of mother attending pediatric out patients department in a tertiary care center. Bangladesh Journal of Child Health. 2014;37(3):138-41. https://doi.org/10.3329/ bjch.v37i3.18616

39. Safar Alizadeh F, Parto Azam H, Sadighi R. Investigating the relationship between the awareness of the mothers referring to health and treatment centers in khoy and their function about the nourishment of the children under 3 years old. J Urmia Nurs Midwifery Fac. 2009;7(3):152-6.

40. Khazaei T, Amoozeshi Z, Ahmadi S, Safamanesh B, Mahmoodi H. The effect of education on mother's knowledge and practiceabout supplementary nutrition for children under one year. Modern Care Journal. 2006;3(3):10-14.

41. Golshiri P, Sharifirad G, Baghernezhad F. Comparison of two methods of education (lecture and self learning) on knowledge and practice of mothers with under 3 year old children about growth monitoring and nutritional development stages. Iranian Journal of Medical Education. 2011;10(5):927-36. 